DE93 006409

\author{
Trim Simulations and Possible Studies \\ for Edge-On Ion Irradiation of Electron Microscope Specimens* \\ Loren J. Thompson, Charles W. Allen, Marcus C. Frischherz,** \\ and Mauro P. Otero*** \\ Materials Science Division \\ Argonne National Laboratory, Argonne, IL 60439-4838, USA \\ **Also Atominstitut der Österreichischen Universitäten \\ Vienna, AUSTRIA \\ ***Also Fundacão de Tecnologia Industrial, \\ Lorena-SP 12600, BRASIL
}

The submitted manuscript has been authored
by a contractor of the U.S. Covernment under
contract No. W-31-109-ENG-38. Accordingly.
the U.S. Governmemt retalns a nonexclusive.
royally-tree license to publish or reproducs the
published form of this contribution, of allow
others to do so, for U.S. Government purpoees.

DISCLAIMER

\begin{abstract}
This report was prepared as an account of work sponsored by an agency of the United States Government. Neither the United States Government nor any agency thereof, nor any of their employees, makes any warranty, express or implied, or assumes any legal liability or responsibility for the accuracy, completeness, or usefulness of any information, apparatus, product, or process disclosed, or represents that its use would not infringe privately owned rights. Reference herein to any specific commercial product, process, or service by trade name, trademark, manufacturer, or otherwise does not necessarily constitute or imply its endorsement, recommendation, or favoring by the United States Government or any agency thereof. The views and opinions of authors expressed herein do not necessarily state or reflect those of the United States Government or any agency thereof.
\end{abstract}

*Work supported by the U.S. Department of Energy, Office of Basic Energy Sciences-Materials Sciences, under Contract No. W-31-109-Eng-38.

Manuscript submitted to the 1992 Fall Meeting of the Materials Research Society, Boston, Massachusetts, November 30-December 4, 1992 


\title{
TRIM SIMULATIONS AND POSSIBLE STUDIES FOR EDGE-ON ION IRRADIATION OF ELECTRON MICROSCOPE SPECIMENS*
}

\author{
LOREN J. THOMPSON, CHARLES W. ALLEN, MARCUS C. FRISCHHERZ** AND \\ MAURO P. OTERO*** \\ Materials Science Division, Arģonne National Laboratory, Argonne, IL 60439 \\ (** Also Atominstitut der Österreichischen Universitäten, Vienna, Austria) \\ (*** Also Fundação de Tecnologia Industrial, Lorena-SP 12600, Brasil)
}

\begin{abstract}
A TRIM code [1] has been modified to simulate a special technique, first described at the Spring 92 MRS Meeting [2], for in situ transmission electron microscope (TEM) experiments involving simultaneous ion irradiation, in which the resultant phenomena are observed as in a cross-section TEM specimen without further specimen preparation. Instead of ion-irradiating the film or foil specimen normal to the major surfaces and observing in plan view (i.e., in essentially the same direction), the specimen is irradiated edge-on (i.e., parallel to the major surfaces) and is observed normal to the depth direction of the irradiation. The results of calculations utilizing the modified TRIM code are presented for cases of 200 and $500 \mathrm{keV}$ Co impinging onto the edge of Si films 200 and $600 \mathrm{~nm}$ thick. The limitations of the technique are discussed and the feasibility of experiments involving implantation of $\mathrm{Co}$ into $\mathrm{Si}$ and the formation of $\mathrm{CoSi}_{2}$, which employ this technique, are briefly discussed.
\end{abstract}

\section{INTRODUCTION}

Common practice when in situ ion irradiation experiments in a TEM are performed is to observe the specimen and hence the resulting phenomena in plan view, the irradiation and viewing directions being more or less normal to the surface of the specimen. Furthermore because of the requirement that TEM specimens must be relatively thin (several tenths of $\mu \mathrm{m}$ and substantially less for heavy metals), deep implantation-type experiments, for example, have not been possible in situ. Rather, following irradiation and usually subsequent heat treatment, a cross-section TEM (XTEM) specimen is prepared in order to analyze the material microstructure. Although quite laborious, this is a very useful and powerful technique for obtaining a lot of information from a single specimen, information which may be directly correlated to other techniques such as Rutherford Backscattering Spectroscopy (RBS). XTEM techniques have largely replaced the much more laborious and oftentimes uncertain "sectioning" techniques involving carefully controlled thinning of bulk irradiated material from the irradiated side to a desired depth, followed by backthinning to produce the electron transparent region corresponding to that depth. Such sectioning techniques required multiple specimens with identical irradiation and thermal histories. In principle at least, the specimen preparation problems associated with the XTEM and other sectioning techniques can be circumvented by performing an in situ TEM experiment in which the specimen is ion irradiated at 90 degrees with respect to the TEM viewing direction; i.e., the ion irradiation is incident on an edge of the specimen, parallel to, rather than normal to, the major surfaces of the specimen. The ideal disposition of the specimen with respect to the electron and ion beams for this situation is depicted in Fig. 1. This turns the specimen into a kind

"Work supported by the U. S. Department of Energy, BES-Materials Sciences, under Contract W-31-109-Eng-38. 


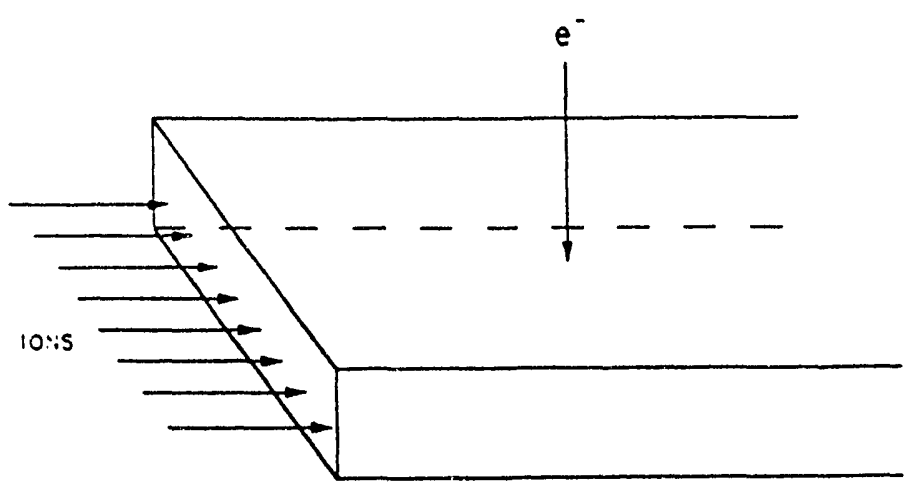

Fig. 1. Ideal configuration for in situ TEM experiment involving edge-on ion irradiation with simultaneous TEM analyses.

of dynamic cross-section specimen as far as the observer is concerned. The advantage of this is that the time evolution of resultant phenomena is available, so that a single specimen serves the purposes of a series of XTEM specimens in a particular irradiation experiment. The chief disadvantage of this edge-on geometry, of course, is that there are two free surfaces in one of the directions which is lateral with respect to the incident ion beam. In order to assess the experimental conditions under which these free surfaces do not dominate the course of resulting phenomena, a conventional TRIM (transport of recoils in metals) simulation has been modified to take account of the finite lateral dimension of the bombarded specimen. The purposes of this paper are to describe this program modification briefly and to present characteristic results of calculations for the edge-on implantation of Co ions into Si films for two ion energies and two specimen thicknesses. Finally, because the simulation assumes no migration of atoms or defects following displacement events, the additional limitations to the proposed experimental technique, arising from the finite mobilities of atoms during real experiments of the deep implantation-type, are assessed.

\section{TRIM SIMULATION}

The simulation of ion irradiation experiments for the geometrical situation depicted in Fig. 1 is based on TRIM 90, the source code for which was supplied by Ziegler [3]. The graphics were stripped from the source code and the program adapted to the Macintosh. The essential adaptation of the program to this problem is threefold. First, one of the lateral dimensions (y-direction), which in standard TRIM is infinite, is restricted to finite values corresponding to the thickness of the TEM specimen. Second, this thin specimen is imagined to be composed of a number of slices normal to the $y$-direction, as shown in Fig. 2; the $x$-direction is the inward normal of the surface onto which the ions are incident. Third, tha ions impinge on the specimen edge at $x=0$, at positions random in $y$. In standara TRIM the numerical output of a simulation ignores, of course, the $y$-distributions of defects and implanted atoms, which are essentially uniform if the material is not finite in $y$, and presents only information with respect to depth $x$ from the surface of incidence. in our problem the $y$-distributions as well as the $x$-distributions are important so that the output has also been modified to yield the $x$-distributions within each of the slices. The results which are presented here are for $y$-slice thicknesses $\Delta y=10$ or $30 \mathrm{~nm}$ and an $x$-direction calculation depth of $1 \mu \mathrm{m}$ ( that is, the calculation increment $\Delta x=10 \mathrm{~nm}$ ). The results for implantation of Co ions of two energies, 0.2 and $0.5 \mathrm{MeV}$, incident on Si films of two thicknesses, 200 and $600 \mathrm{~nm}$, are discussed. Because the number of $y$-slices in both cases is 20, the slice thickness $\Delta y=10 \mathrm{~nm}$ for the $200 \mathrm{~nm}$ thick film and $30 \mathrm{~nm}$ for the $600 \mathrm{~nm}$ film. Each simulation involves a total of 100,000 ions incident on the 


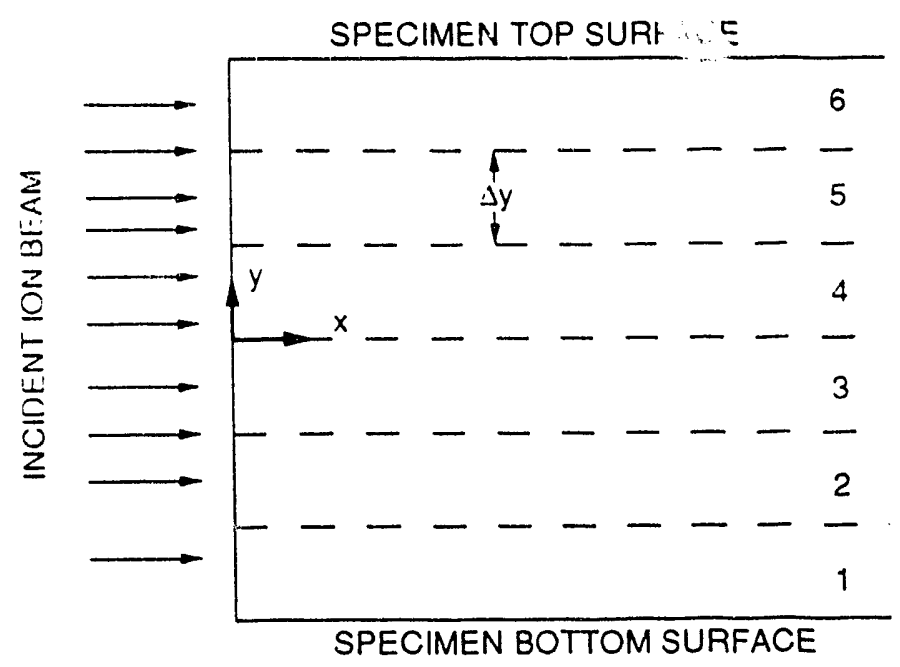

Fig. 2. Thin film with ion beam incident onto edge of film. For computational purposes, film is divided into slices, $\Delta y$ thick, normal to $y$-direction. Ion incidence is random with respect to $y$.

specimen edge at. $x=0$ (random incidence with respect to $y$ ). With a Mac IIFX, the machine times for the implantation calculations range from about 11 hours for 200 $\mathrm{keV}$ Co ions on the edge of a $200 \mathrm{~nm}$ Si film to about 36 hours for $1500 \mathrm{keV}$ ions on a $600 \mathrm{~nm}$ film. In these calculations, no account has been taken of sputtering of the front surface, which can overwhelm other effects during low temperature implantation [4].

After verifying that calculated results are the same within statistical limits for corresponding $y$-slices equidistant from the center of the film (for example, the pairs 1-6, 2-5 and 3-4 in Fig. 2), the output results of the distributions of implanted Co for each of these $y$-slice pairs are summed. The results are normalized so that the graphical output shows Co ions implanted (per 100,000 Co ions incident on the edge of the specimen) within a given $y$-slice, pair per increment $\Delta x$ as a function of $x$.

At the present time the $y$-slice thickness $\Delta y$ is not an independent input parameter of the modified TRIM program, but is the specimen thickness $t$ divided by 20 ; i.e., there are always $20 \mathrm{y}$-slices. The main reason for this seemingly arbitrary choice is the limited capacity of the Mac II to accommodate a significantly larger amount of output data, the amount increasing in proportion to the number of $y$-slices.

The implantation results from the TRIM calculations for the two incident ion energies and two film thicknesses are presented in Fig. 3. Each graph includes the implanted Co distributions for all ten of the $y$-slice pairs, that for the outermost pair (slices 1 and 20) being the lowest curve in each case. The quantity $I$ is the fraction of ions implanted in the film.

\section{$200 \mathrm{keV}$ Co on Edge of $200 \mathrm{~nm}$ Si Film (Figure 3a)}

The $y$-slice thickness $\Delta y=10 \mathrm{~nm}$. Clearly the $y$-slices at the surfaces (lowest curve) have retained less than half as much $C_{0}$ as the innermost $y$-slices. The behavior of the first $30 \mathrm{~nm}$ from each surface is noticeably different from the remaining 70 percent of the film. However, of the total number of ions incident on the film edge, 89 percent have been implanted.

\section{$200 \mathrm{keV}$ Co on Edge of $600 \mathrm{~nm}$ Si Film (Figure 3b)}

The $y$-slice thickness $\Delta y=30 \mathrm{~nm}$. The distribution of implanted Co in the two $y$-slices nearest the surfaces are easily distinguished from the distributions in the other $y$ slices. Note that these two y-slice pairs in this case represent a total of $120 \mathrm{~nm}$ of the 


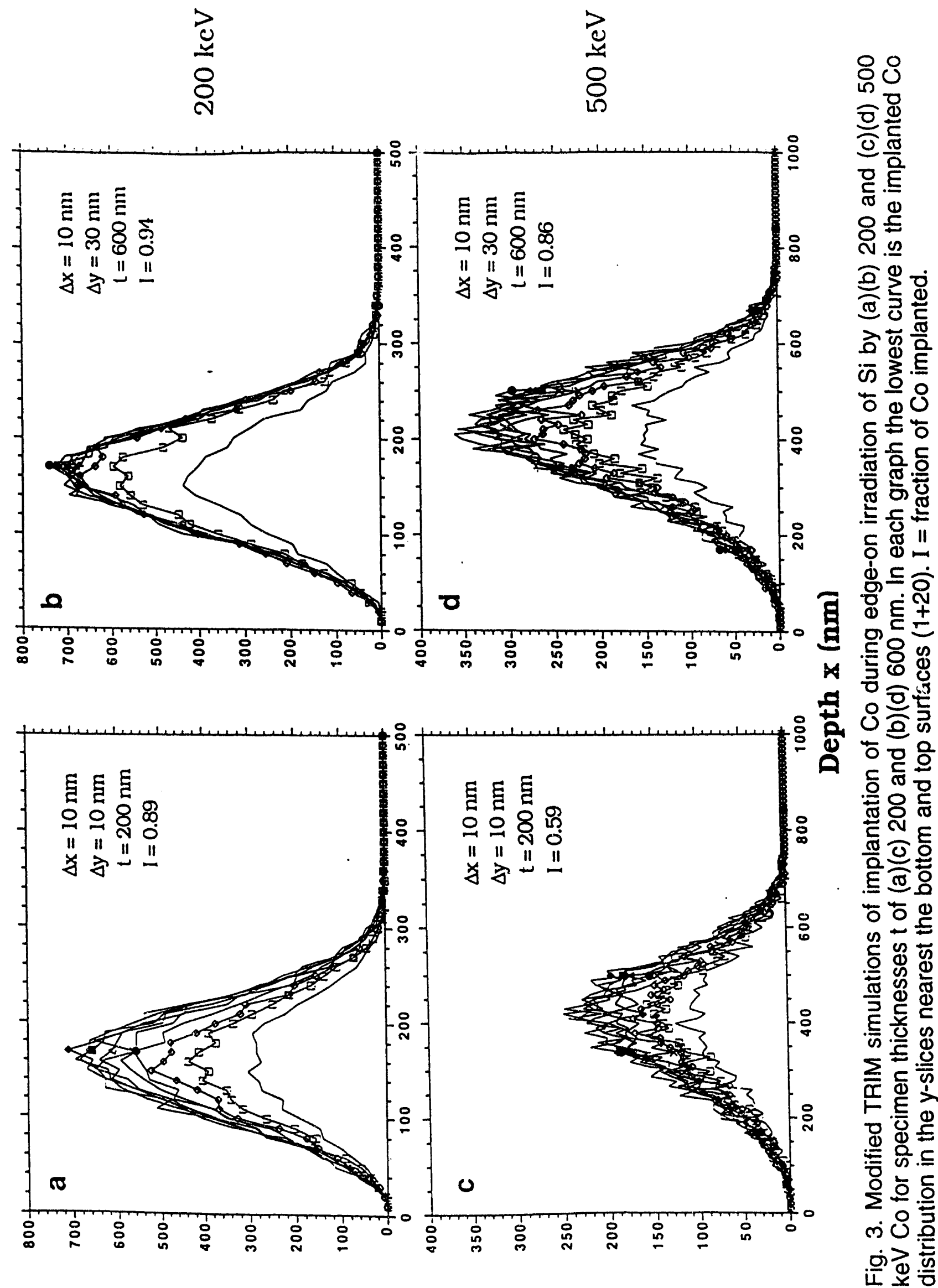


film so that the Co distributions in 80 percent of the film are virtually identical. In this thicker film 94 percent of the incident Co has been retained.

\section{$500 \mathrm{keV}$ Co on Edge of $200 \mathrm{~nm}$ Si Film (Figure $3 \mathrm{c}$ )}

The $y$-slice thickness $\Delta y=10 \mathrm{~nm}$. Clearly the $y$-slices at the surfaces (lowest curve) have again retained less than half as much $C o$ as the inner $y$-slices. The behavior of the first $30 \mathrm{~nm}$ from each surface is noticeably different from the remaining 70 percent of the film. Of the total number of ions incident on the film edge, only 59 percent have been implanted.

\section{$500 \mathrm{keV}$ Co on Edge of $600 \mathrm{~nm}$ Si Film (Figure 3d)}

The $y$-slice thickness $\Delta y=30 \mathrm{~nm}$. The distribution of implanted Co in the three $y$ slices nearest the surfaces are easily distinguished from the distributions in the other $y$-slices. Note that these three y-slice pairs in this case represent a total of $180 \mathrm{~nm}$ of the film, which is almost the thickness of the Si film described in Figure 3a, consistent with the magnitudes of the numbers of Co atoms implanted. In this thicker film, however, 86 percent of the incident $C o$ has been retained.

There is very little, if any, difference between the implanted Co distributions within the inner y-slices corresponding to Figs. 3(a), (b) and (d) and those from standard TRIM calculations. There is a significant difference for $500 \mathrm{keV}$ Co on a $200 \mathrm{~nm}$ film of Si, however, as is clear from a comparison of Figs. 3(c) and (d).

\section{FEASIBILITY OF SUCCESS OF EDGE-ON IMPLANTATION EXPERIMENTS}

The implantation of $\mathrm{Co}$ into bulk Si has been widely studied by both RBS and XTEM [4-8]. There still remain questions, nevertheless, regarding the specific sequence of events during the formation, ripening and ultimate coalescence of $\mathrm{CoSi}_{2}$ precipitate crystals. Such implantations must be performed at an elevated temperature such as $620 \mathrm{~K}$ or higher in order to prevent amorphization of the Si. Furthermore the development of the silicide is usually accomplished by subsequent annealing at still higher temperatures rather than implanting at such temperatures $(850-1400 \mathrm{~K}$, for example). Co is a fast diffuser in Si [9]. Ref. 9 does not present data for Co, but on the assumption that the diffusivity is similar to that for $\mathrm{Cu}$ and $\mathrm{Ni}$, one would conclude that at $700 \mathrm{~K}$, for instance, the diffusivity is $10^{-10}$ to $10^{-11} \mathrm{~m}^{2} \mathrm{~s}^{-1}$. For implantations requiring one hour, one would estimate that Co implanted early in the process at 700 $\mathrm{K}$ could migrate roughly $1 \mathrm{~mm}$ in one hour if in solid solution. In fact, of course, CoSi layers form at reasonable implant depths, suggesting that nucleation of the silicide readily occurs as a consequence of the very low equilibrium solubility of $\mathrm{Co}$ in $\mathrm{Si}$, thus immobilizing the Co. An alternative explanation comes from Hashimoto et al. [10], whose diffusivity data for $\mathrm{Co}$ in $\mathrm{Si}$ indicate that $\mathrm{Co}$ is a fast diffuser only at sufficiently high temperatures, such as $1200 \mathrm{~K}$ and above, but the activation enthalpy is much greater than those of regular fast diffusers such as $\mathrm{Cu}, \mathrm{Ni}, \mathrm{Li}$ and $\mathrm{Fe}$ [9] and $D_{0}$ is anomalously large. Consequently the diffusivity of $C_{0}$, obtained by a long extrapolation of the high temperature data to $700 \mathrm{~K}$, yields a diffusivity of the order $10^{-20} \mathrm{~m}^{2} \mathrm{~s}^{-1}$. This would reduce the diffusion distances, as estimated above, by 5 orders of magnitude or more and clearly changes the complexion of the problem. The edge-on implantation of $\mathrm{Co}$ into a Si film seems worth the effort, therefore, especially for a relatively thick film (e.g., $600 \mathrm{~nm}$ ) and incident ion energy of $500 \mathrm{keV}$ or less. Such an in situ experiment can be readily performed in the HVEM at Argonne's HVEM-Tandem Facility, with which the microstructural evolution can be followed by intermittent observation, employing $1 \mathrm{MeV}$ electrons in conjunction with 
the relatively thick Si film (intermittent in order to avoid any substantial electron damage). Higher energy implantation may also be suitable, but the distribution of implanted Co will be less uniform across the film thickness and the implantation efficiency (the fraction implanted I in Fig. 3) is decreased. Results of such an edgeon in situ ion irradiation experiment involving the partial amorphization of Si by 1.0$1.5 \mathrm{MeV} \mathrm{Kr}$ bombardment have been discussed previously [2].

\section{OTHER OUTPUT OF MODIFIED TRIM SIMULATIONS}

In addition to the distribution of ion ranges presented here, the modified TRIM program will compute all the usual quantities of TRIM 90, including damage distributions. The calculation of the distribution of ion ranges presented above is relatively rapid because only the ion trajectory is determined. On the other hand, the calculation of damage distribution in each slice is more time-consuming; for example, for ten thousand 1.5 MeV Co ions on a $600 \mathrm{~nm}$ thick Si film, which is sufficient for reasonable statistics, the computational time for a Mac IIFX is about 48 hours. In general, especially for higher energy implants, the distributions of damage are less uniform across the film thickness for a given depth than are the implanted ion distributions. It might prove interesting to utilize this difference to examine the effects of different damage rates for a given implantation rate.

\section{ACKNOWLEDGMENTS}

We wish to express our gratitude to Dr. Ziegler for supplying the source code for TRIM 90 and to Dr. Wolfgang Jäger for helpful discussions regarding implantation experiments of $\mathrm{Co}$ into $\mathrm{Si}$.

\section{REFERENCES}

[1] J. F. Ziegler, J. P. Biersack and U. Littmark, in The Stopping and Range of lons in Solids, ed. J. F. Ziegler (Pergamon, New York, 1985).

[2] M. P. Otero and C. W. Allen, Mat. Res. Soc. Symp. Proc. 268 (1992) 337.

[3] J.F. Ziegler, private communication.

[4] A. E. White, K. T. Short, R. C. Dynes, J. P. Garno and J. M. Gibson, Appl. Phys. Lett. 50 (1987) 95-97.

[5] A. E. White, K. T. Short, R. C. Dynes, J. M. Gibson and R. Hull, Mat. Res. Soc. Symp. Proc. 100 (1988) 3-15.

[6] A. H. Van Ommen, C. W. T. Bulle-Lieuwma, J. J. M. Ottenheim and A. M. L. Theunissen, J. Appl. Phys. 67 (1990) 1767-1778.

[7] P. F. P. Fichtner, W. Jäger, K. Radermacher and S. Mantl, Nucl. Instrum. Methods B59/60 (1991) 632-636.

[8] R. Jebasinski, S. Mantl, K. Radermacher, P. Fichtner, W. Jäger and Ch. Buchal, Mat. Res. Soc. Symp. Proc. 201 (1991) 411-416.

[9] W. Frank, U. Gösele, H. Mehrer and A. Seeger, in Diffusion in Crystalline Solids, ed. by G. E. Murch and A. S. Nowick.(Academic Press, New York, 1984).

[10] K. Hashimoto, H. Nakashima and K. Hashimoto, Japan. J. Appl. Phys. 27 (1988) 1776. 

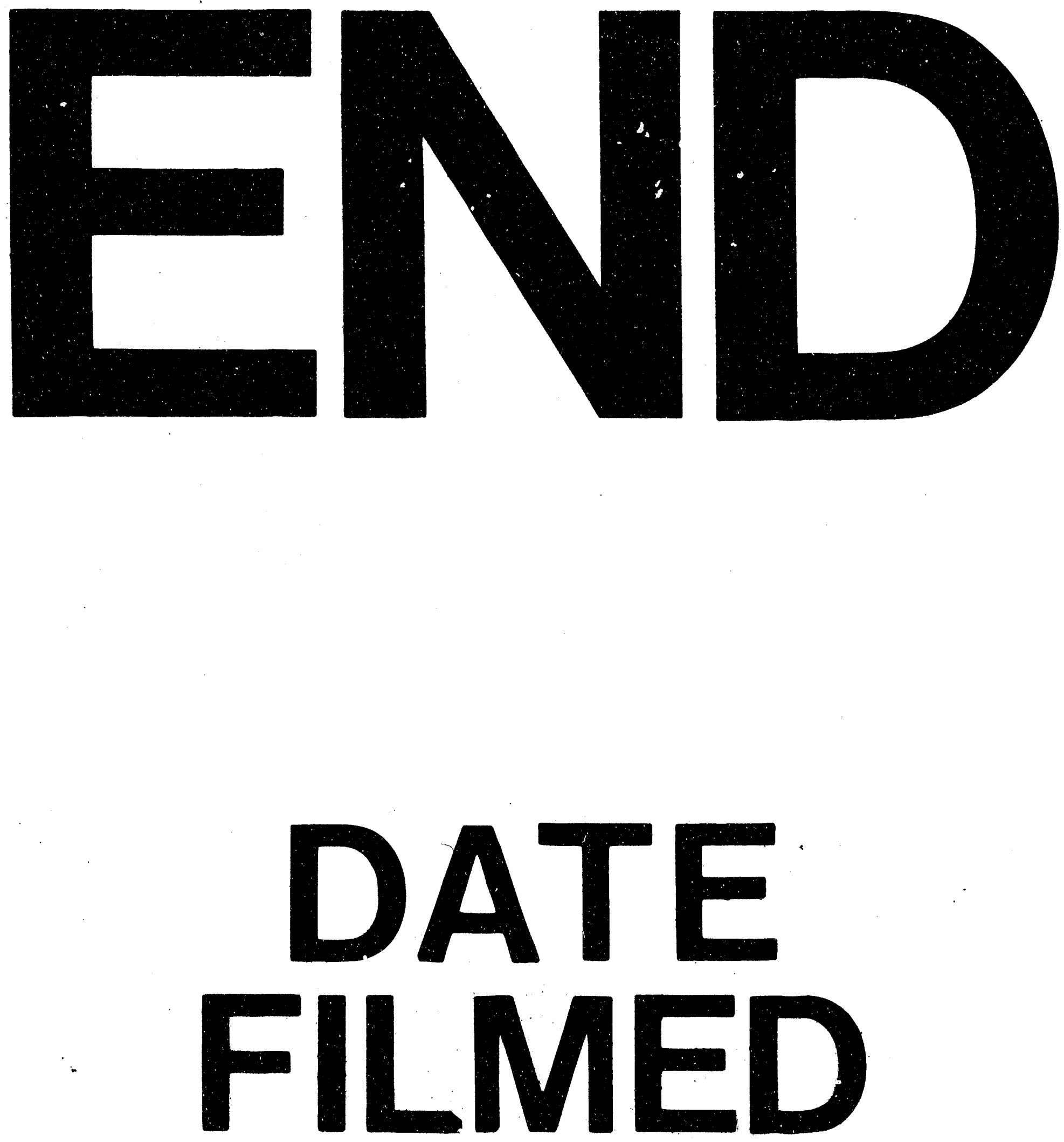

1

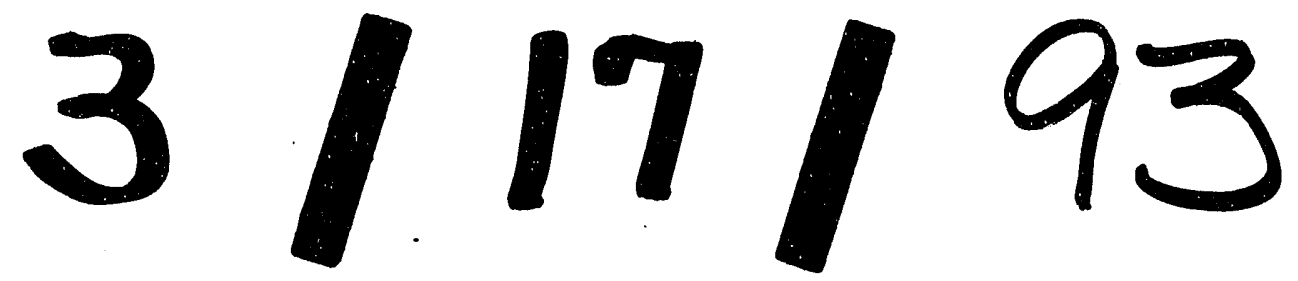


